\title{
Checklist of periphytic diatoms in streams of the Pirapó River basin, Paraná state, Brazil
}

\author{
Carina Moresco $^{1 *}$ and Liliana Rodrigues ${ }^{2}$ \\ 1 Faculdade Integrado de Campo Mourão, Rodovia BR 158, KM 207, CEP 87.300.970, Campo Mourão, PR, Brazil \\ 2 Universidade Estadual de Maringá. Avenida Colombo, 5790, 87020-900, Maringá, PR, Brazil \\ * Corresponding author. E-mail: morescocarina@gmail.com
}

\begin{abstract}
In lotic environments, diatoms have high richness and represent important elements of biodiversity and genetic resources of these sites. Given the impacts caused by urbanization and agriculture on streams and the responses of the periphytic diatom community, this study aims to provide a checklist of epilithic diatoms in two streams: one located in an urban area and the other in rural area. Bimonthly samples were taken along the longitudinal gradient of streams (headwaters, middle and mouth) from July 2007 through August 2008. Permanent slides were deposited in the Herbarium of the State University of Maringá. In the rural stream, we identified 124 species belonging to 47 genera and 26 families and in the urban stream, 79 species belonging to 34 genera and 20 families. The streams had 68 taxa in common. In both streams, the most representative family was Naviculaceae.
\end{abstract}

Key words: Bacillariophyceae; algae; urban stream; epilithon; water quality; bioindicator

\section{INTRODUCTION}

In rivers and streams, diatoms are among the communities with the highest number of species and represent important elements of biodiversity and genetic resources of these sites (Patrick 1961). This group may have significant biomass (Wehr and Sheath 2003; Azim and Asaeda 2005), contributing to the primary productivity of these ecosystems (Pan et al. 1999). Furthermore, diatoms have been used as biological indicators of water quality in many countries (Whitton and Kelly 1995; Kelly et al. 1998; Gómez and Licursi 2001; Ács et al. 2005; Potapova and Charles 2007; Delgado et al. 2012). In Brazil, most studies on this community are concentrated in the south. Much of the information about the diatom flora in lotic environments of the Paraná state is found in masterss theses (Brassac and Ludwig 2003). Considering the impacts caused by urbanization and agriculture on streams and the responses of the periphytic diatom community (Moresco and Rodrigues 2014), this study aims to provide a checklist of epilithic diatoms in two streams: one located in an urban area and the other in rural area.

\section{MATERIALS AND METHODS Study site}

The Pirapó River basin is part of the large system of the Paraná River and it is an important left bank tributary (south bank) of the Paranapanema River (Maack 2002). This physiographic region is called the Third Plateau of Paraná, specifically in the polygon bounded by the longitudes $51^{\circ} 15^{\prime}$ and $52^{\circ} 15^{\prime} \mathrm{W}$ and latitudes $22^{\circ} 30^{\prime}$ and $23^{\circ} 30^{\prime} \mathrm{S}$, with a drainage area of approximately 5,076 $\mathrm{km}^{2}$. The climate in the region is subtropical, with average temperatures above $20^{\circ} \mathrm{C}$. This basin has great importance in relation to abstraction of water for supply, development of agricultural activities and ecological tourism in most cities in the region (Peron et al. 2009).

The municipality of Maringá is located on the interfluve of Pirapó and Ivaí river basins and encompasses several springs in the urban area. The region is relatively industrialized and urbanized, and the city of Maringá is the most important urban center of the region, with about 390,000 inhabitants.

The microbasins of the sampled streams have different land use; the course of the Nazare stream is entirely within the urban area, with residential occupation, and receives stormwater and sewage (Moresco and Rodrigues 2014). According to Moresco and Rodrigues (2014), the Remo stream is totally rural, with rotation of crops (corn, soybeans and wheat) along its course (Figure 1).

\section{Data collection}

Bimonthly collections were conducted at three sampling sites (headwaters, middle and mouth) of the streams Nazaré and Remo from July 2007 through August 2008. 


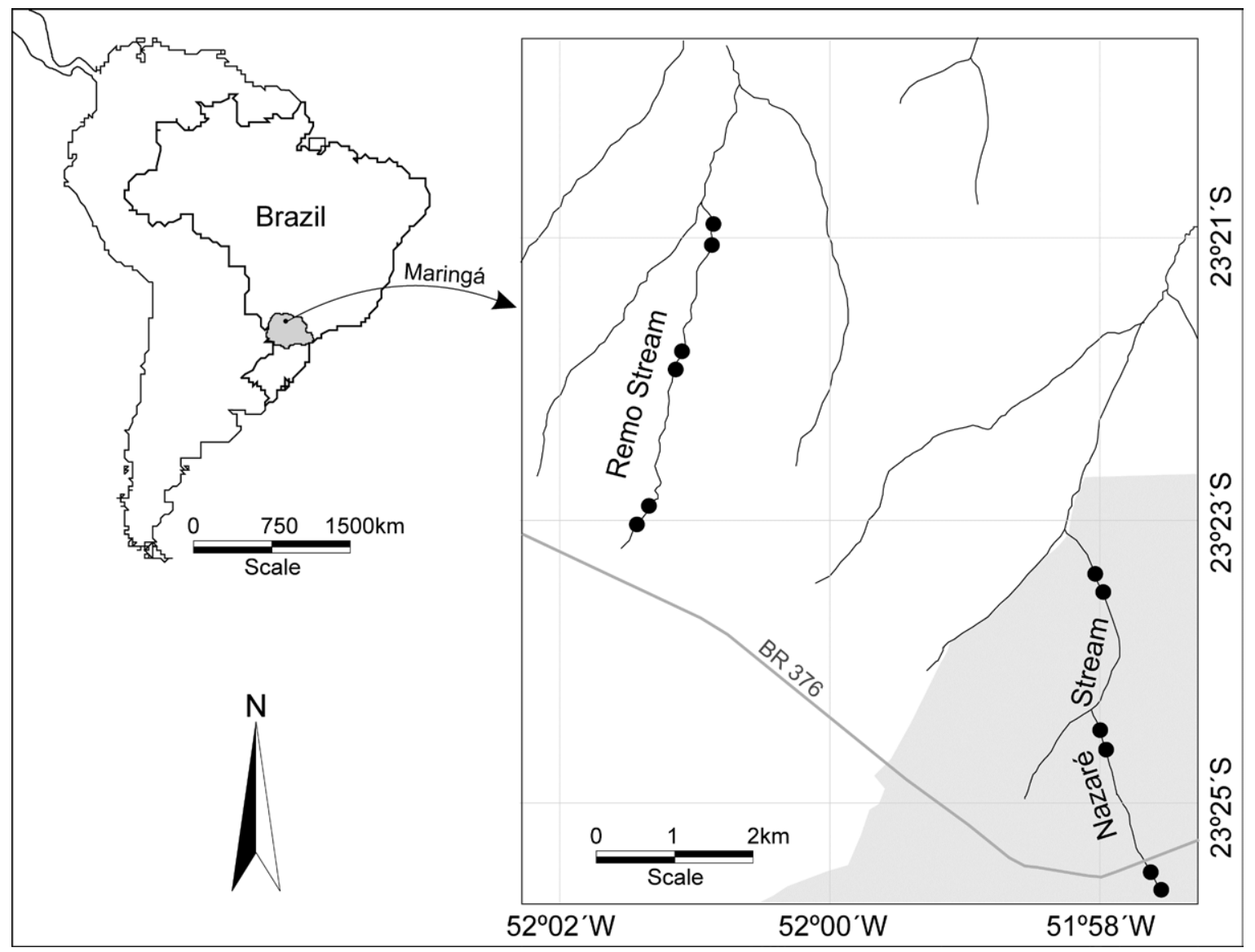

Figure 1. Location of the urban stream and the rural stream in Maringá Municipality, Paraná state, Brazil. White: rural area and grey: urban area.

For the qualitative analysis of the diatom community, we sampled three pebbles at each site, and the average of the total area scraped was $50 \mathrm{~cm}^{2}$. The choice of the substrate considered its abundance and frequency in all sampling sites. The surface opposite to the current flow was scraped with the aid of a brush and razor blade and the material collected was fixed in $4 \%$ formalin (1: 1 ratio). We measured the area scraped with a caliper. The material was oxidized with $\mathrm{KMnO}_{4}$ and $\mathrm{HCl}$ according to the technique proposed by Simonsen (1974), modified by MoreiraFilho and Valente-Moreira (1981). Hyrax was used for mounting the permanent slides. The slides were deposited in the Herbarium of the State University of Maringá (HUEM 16508 to 16543). Diatoms were collected under license of Instituto Brasileiro do Meio Ambiente e dos Recursos Naturais Renováveis IBAMA process 02040.000093/06-45.

Individuals were identified under an optical microscope Olympus $\mathrm{CX}_{31}$ using specialized taxonomic literature (Patrick and Reimer 1966; Krammer and Lange-Bertalot 1986, 1988, 1991a, 1991b; Lange-Bertalot 2001; Krammer 2002; Metzeltin and Lange-Bertalot 1998, 2007; Metzeltin et al. 2005). The taxonomic classification used in this study followed the proposal of Round et al. (1990).

\section{RESULTS}

In the rural stream, 124 species were found, belonging to 47 genera and 26 families (Table 1). In the urban stream, 79 species were identified, belonging to 34 genera and 20 families. The streams had 68 (54.83\%) taxa in common. The rural stream had $56(45.16 \%)$ exclusive species and the urban stream only 11 (8.87\%) exclusive species. In total, 135 species were identified.

In the rural environment, the most species-rich family was Naviculaceae (25 spp.), followed by Cymbellaceae (12 spp.), Bacillariaceae and Pinnulariaceae (10 spp. each), Sellaphoraceae (8 spp.), Gomphonemataceae (7 spp.), Diadesmidaceae (6 spp.). The other families presented five species or less (Eunotiaceae ( 5 spp.), Achnanthaceae, Amphipleuraceae and Surirellaceae (4 spp. each), Alaucoseiraceae, Fragillariaceae, Catenulaceae, Pleurosigmataceae and Stauroneidaceae (3 spp. each), Achanthidiaceae, Brachysiraceae, Cocconeidaceae and Diploneidaceae (2 spp. each) and Melosiraceae, Orthoseiraceae, Stephanodiscaceae, Triceratiaceae, Neidiaceae and Rhopalodiaceae (1 sp. each).

Regarding the urban stream, the family with the highest number of species identified was Naviculaceae ( 16 spp.), followed by Bacillariaceae (10 spp.), Pinnulariaceae (8 spp.), Cymbellaceae (7 spp.), Gomphonemathaceae (6 spp.), and Diadesmidadeceae and Sellaphoraceae (5 spp. 
Table 1. Taxa of Bacillariophyta recorded in the R (rural) and U (urban) streams, municipality of Maringá, Paraná state. Periods 1 (July 2007 ), 2 (September 2007), 3 (December 2007), 4 (February 2008), 5 (April 2008), 6 (May-June 2008).

\begin{tabular}{|c|c|c|c|c|c|c|c|c|c|c|c|c|}
\hline Taxa & $\mathbf{R 1}$ & $\mathbf{R 2}$ & $\mathbf{R 3}$ & R4 & $\mathbf{R 5}$ & R6 & U1 & U2 & U3 & U4 & U5 & U6 \\
\hline \multicolumn{13}{|l|}{ COSCINODISCOPHYCEAE } \\
\hline \multicolumn{13}{|l|}{ Alaucoseiraceae } \\
\hline Aulacoseira ambigua (Grunow) Simonsen & & & & & $x$ & $\mathrm{x}$ & & & & & & \\
\hline Aulacoseira granulata (Ehrenberg) Simonsen & & & & $\mathrm{x}$ & & $x$ & & & & & & \\
\hline Aulacoseira sp. & & & & & $x$ & $x$ & & $x$ & & & & \\
\hline \multicolumn{13}{|l|}{ Melosiraceae } \\
\hline Melosira varians Agardh & & & & & $x$ & $x$ & & & & & & \\
\hline \multicolumn{13}{|l|}{ Orthoseiraceae } \\
\hline Orthoseira sp. & & & & & $x$ & & & & & & & \\
\hline \multicolumn{13}{|l|}{ Stephanodiscaceae } \\
\hline Cyclotella meneghiniana Kützing & & $x$ & & & $\mathrm{x}$ & & & & $x$ & $x$ & $x$ & $x$ \\
\hline \multicolumn{13}{|l|}{ Triceratiaceae } \\
\hline Pleurosira laevis (Ehrenberg) Compère & & $\mathrm{x}$ & & & $\mathrm{x}$ & & & & & & & \\
\hline \multicolumn{13}{|l|}{ FRAGILARIOPHYCEAE } \\
\hline Fragilaria rumpens (Kützing) Carlson & $\mathrm{x}$ & $\mathrm{x}$ & $x$ & & $\mathrm{x}$ & $\mathrm{x}$ & & & & & & \\
\hline Synedra rumpens Kützing var. familiaris (Kützing) Grunow & $x$ & & & & & & & & & & & \\
\hline Ulnaria ulna (Nitzsch) Compère & $x$ & $x$ & $x$ & $x$ & $x$ & $x$ & & & $x$ & $x$ & $x$ & \\
\hline
\end{tabular}

\section{BACILLARIOPHYCEAE}

\section{Achnanthaceae}

Achnanthes exigua Grunow

Achnanthes hungarica Grunow

Achnanthes inflata Grunow

Achnanthes lanceolata (Brébisson ex Kützing) Grunow

Achnanthes rupestoides Hohn

\section{Achnanthidiaceae}

Achnanthidium minutissimum (Kützing) Czarnecki

Planothidium conspicuum (Mayer) Aboal in Aboal

\section{Amphipleuraceae}

Amphipleura lindheimeriGrunow

Frustulia crassinervia (Brébisson) Lange-Bertalot \& Krammer

Frustulia pumilio Lange-Bertalot \& Rumrich

Frustulia vulgaris (Thwaites) De Toni

\section{Bacillariaceae}

Hantzschia amphioxys (Ehrenberg) Grunow

Nitzschia amphibia Grunow

Nitzschia clausii Hantzsch

Nitzschia frustulum (Kützing) Grunow

Nitzschia gandersheimiensis Krasske

Nitzschia gracilis Hantzsch

Nitzschia cf. inconspicua Grunow

Nitzschia linearis Smith

Nitzschia lorenziana Grunow

Nitzschia palea (Kützing) Smith

Tryblionella debilis Arnot

Tryblionella levidensis Smith

\section{Brachysiraceae}

Brachysira neoexilis Lange-Bertalot

Brachysira sp.

\section{Catenulaceae}

Amphora copulata (Kützing) Schoeman \& Archibald

Amphora montana Krasske

Amphora normannii Rabenhorst

\section{Cocconeidaceae}

Cocconeis placentula var. acuta Meister

Cocconeis placentula var. euglypta (Ehrenberg) Cleve

\section{Cymbellaceae}

Cymbella microcephala Grunow

Cymbella naviculiformis Auerswaldex Heiberg

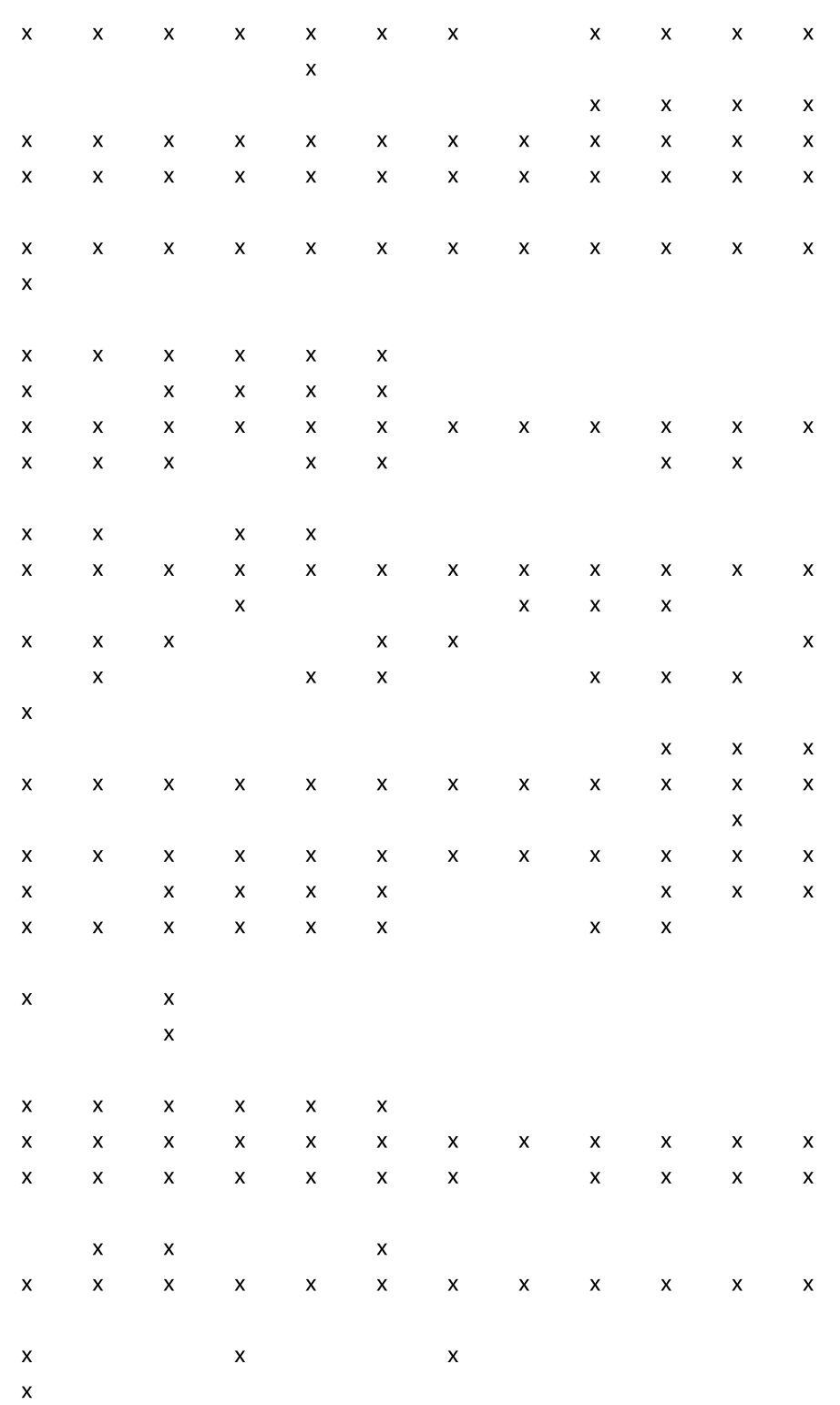

Continued 
Table 1. Continued.

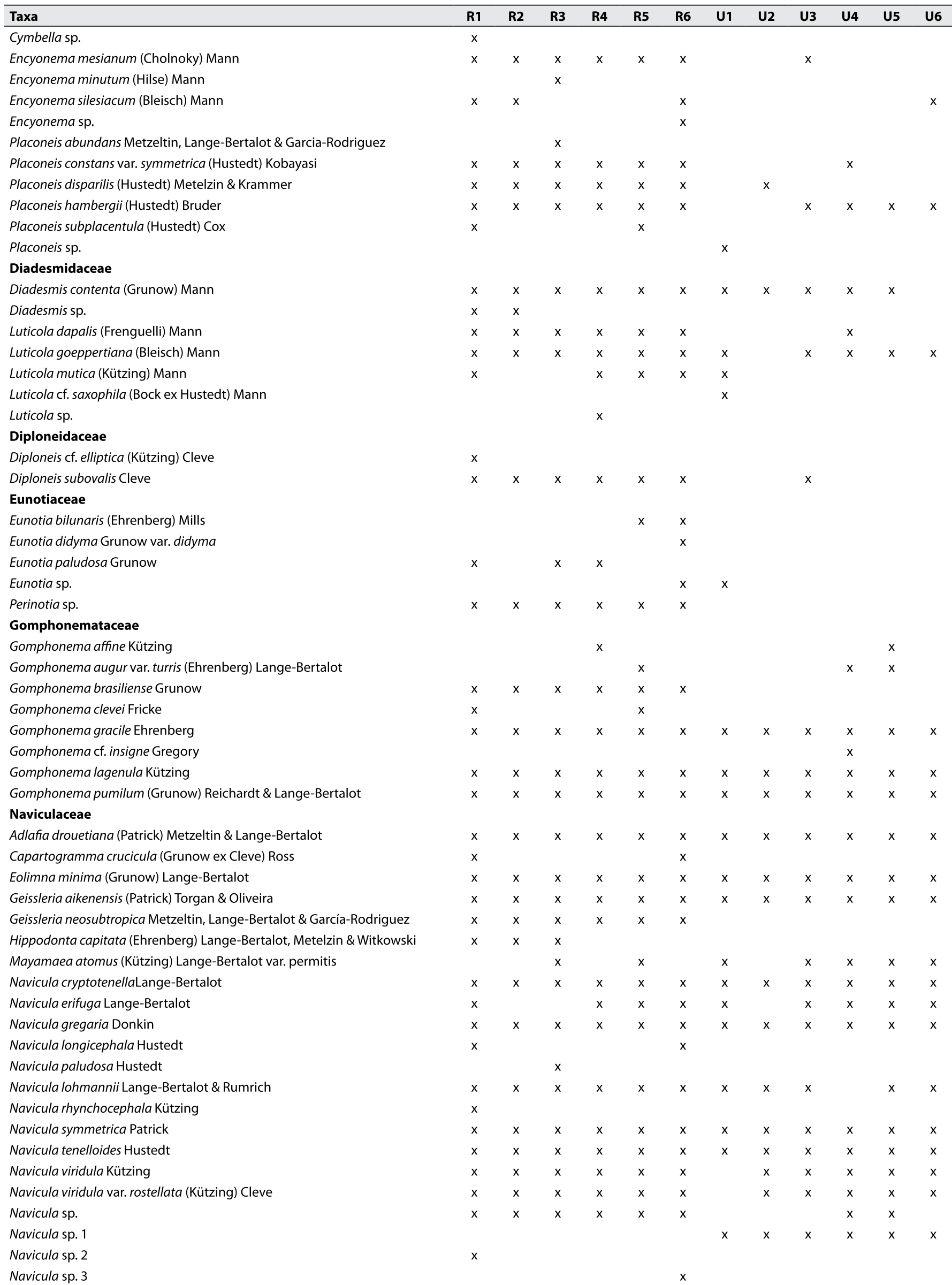


Table 1. Continued.

\begin{tabular}{|c|c|c|c|c|c|c|c|c|c|c|c|c|}
\hline Taxa & $\mathbf{R 1}$ & $\mathbf{R 2}$ & R3 & R4 & R5 & R6 & U1 & U2 & U3 & U4 & U5 & U6 \\
\hline Navicula sp. 4 & & & & $\mathrm{x}$ & $\mathrm{x}$ & $x$ & & & & & & \\
\hline Navicula sp. 5 & & $x$ & & & & & & & & & & \\
\hline Nupela praecipua (Reichardt) Reichardt & $x$ & $x$ & $x$ & $x$ & $x$ & $x$ & $x$ & & $x$ & $x$ & $x$ & \\
\hline Nupela sp. & $\mathrm{x}$ & $x$ & $x$ & $x$ & $x$ & $\mathrm{x}$ & $x$ & $x$ & $x$ & $x$ & $x$ & $x$ \\
\hline \multicolumn{13}{|l|}{ Neidiaceae } \\
\hline Neidium affine (Ehrenberg) Pfitzer & & & & & & & & & $x$ & & & \\
\hline Neidium sp. & $x$ & $\mathrm{x}$ & & & & & & & & & & \\
\hline \multicolumn{13}{|l|}{ Pinnulariaceae } \\
\hline Caloneis bacillum (Grunow) Cleve & $x$ & $\mathrm{x}$ & & & $x$ & & $x$ & $x$ & $x$ & $x$ & $x$ & $\mathrm{x}$ \\
\hline Pinnularia doehringii Frenguelli & $x$ & $x$ & $x$ & & $x$ & & & $x$ & $\mathrm{x}$ & $\mathrm{x}$ & $x$ & \\
\hline Pinnularia dubitabilis (Hustedt) Hustedt & & & & & & $x$ & & & & & & \\
\hline Pinnularia gibba (Ehrenberg) Ehrenberg & $x$ & & $x$ & $x$ & $x$ & $x$ & $x$ & $x$ & $x$ & $x$ & & $x$ \\
\hline Pinnularia latarea Krammer & $x$ & $x$ & & & $x$ & $x$ & $x$ & $x$ & $\mathrm{x}$ & $x$ & $x$ & \\
\hline Pinnularia mesolepta (Ehrenberg) Smith & & $x$ & $x$ & $x$ & & & & & & $x$ & & \\
\hline Pinnularia obscura Krasske & & $x$ & & $x$ & & $\mathrm{x}$ & & & & $x$ & & $x$ \\
\hline Pinnularia subcapitata Gregory & $x$ & & & & & & & & & & & \\
\hline Pinnularia viridis (Nitzsch) Ehrenberg & & & & & $x$ & & & & & $x$ & & \\
\hline Pinnularia sp. & & & $\mathrm{x}$ & $x$ & $\mathrm{x}$ & $\mathrm{x}$ & & & & $\mathrm{x}$ & & \\
\hline \multicolumn{13}{|l|}{ Pleurosigmataceae } \\
\hline Gyrosigma acuminatum (Kützing) Rabenhorst & $\mathrm{x}$ & & $x$ & $x$ & $\mathrm{x}$ & $\mathrm{x}$ & & & & $x$ & & \\
\hline Gyrosigma nodiferum (Grunow) Reimer & $\mathrm{x}$ & & & & $\mathrm{x}$ & $x$ & & & & & & \\
\hline Gyrosigma scalproides (Rabenhorst) Cleve & $\mathrm{x}$ & $\mathrm{x}$ & $x$ & & $x$ & $x$ & $x$ & & & & & \\
\hline \multicolumn{13}{|l|}{ Rhopalodiaceae } \\
\hline Rhopalodia gibberula var. vanheurckii (Van Heurck) Müller & $x$ & & & & & & & & & & & \\
\hline \multicolumn{13}{|l|}{ Sellaphoracea } \\
\hline Fallacia ecuadoriana Lange-Bertalot \& Rumrich & $\mathrm{x}$ & $\mathrm{x}$ & $\mathrm{x}$ & $x$ & $x$ & $\mathrm{x}$ & & & & & $\mathrm{x}$ & \\
\hline Fallacia insociabilis (Krasske) Mann & $x$ & $x$ & $x$ & $\mathrm{x}$ & $x$ & $x$ & & & & & & \\
\hline Fallacia monoculata (Hustedt) Mann & $x$ & & & $x$ & & & $\mathrm{x}$ & $x$ & $\mathrm{x}$ & $x$ & $\mathrm{x}$ & $x$ \\
\hline Sellaphora bacillum (Ehrenberg) Mann & & & & & $x$ & & & & & & & \\
\hline Sellaphora pupula (Kützing) Mereschkovsky & $\mathrm{x}$ & $\mathrm{x}$ & $x$ & $\mathrm{x}$ & $\mathrm{x}$ & $\mathrm{x}$ & $\mathrm{x}$ & $x$ & $x$ & $x$ & $x$ & \\
\hline Sellaphora seminulum (Grunow) Mann & $x$ & $x$ & $x$ & $x$ & $x$ & $x$ & $x$ & $x$ & $x$ & $x$ & $x$ & $x$ \\
\hline Sellaphora sp. & $\mathrm{x}$ & & & & & & & & & & & \\
\hline Sellaphora sp. 1 & $x$ & $x$ & $x$ & & $x$ & $\mathrm{x}$ & & $x$ & & $x$ & $x$ & \\
\hline \multicolumn{13}{|l|}{ Stauroneidaceae } \\
\hline Craticula ambigua (Ehrenberg) Mann & & & & & $x$ & $\mathrm{x}$ & & & & & & \\
\hline Craticula submolesta (Hustedt) Lange-Bertalot & & & & & & & & $x$ & & & & \\
\hline Craticula sp. & & & & & & & $x$ & $x$ & $x$ & $\mathrm{x}$ & $x$ & $\mathrm{x}$ \\
\hline Stauroneis cf. kriegeri Patrick & $x$ & $x$ & $x$ & $x$ & $x$ & $x$ & & & & & & $x$ \\
\hline Stauroneis nana Hustedt & $x$ & $x$ & & & & $x$ & & & & & & \\
\hline \multicolumn{13}{|l|}{ Surirellaceae } \\
\hline Stenopterobia schweickerdtii (Cholnoky) Brassac, Ludwig \& Torgan & $\mathrm{x}$ & $\mathrm{x}$ & & & $\mathrm{x}$ & $\mathrm{x}$ & & & & & & \\
\hline Stenopterobia sp. & $\mathrm{x}$ & $x$ & $x$ & $x$ & $\mathrm{x}$ & $x$ & $\mathrm{x}$ & $x$ & $\mathrm{x}$ & $x$ & $x$ & $x$ \\
\hline Surirella linearis Smith & & & & & & $x$ & & & & & & \\
\hline Surirella stalagma Hohn \& Hellerman & & & & & & $\mathrm{x}$ & & & & & & \\
\hline Surirella cf. tenera Gregory & & & & & & & & & & $x$ & & \\
\hline
\end{tabular}

each), Achnanthaceae (4 spp.), Stauroneidaceae (3 spp.), Amphipleuraceae, Catenulaceae, Pleurosigmataceae and Surirellaceae (2 spp. each), Aulacoseiraceae, Stephanodiscaceae, Fragilariaceae, Achnanthidiaceae, Cocconeidaceae, Diploneidaceae and Neidiaceae (1 sp. each) (Table 1). Some of the species found are shown in Figure 2.

\section{DISCUSSION}

The assemblages of the periphytic diatoms studied were different between the urban stream and the rural stream. Studies on streams draining urban centers have shown a decline in the diatom richness associated with organic pollution (Lobo et al. 1995; Sonneman et al. 2001).
While both agriculture and urbanization cause impacts on rivers and streams, the pollution derived from urban areas is more intense than that registered in rural areas (Odum 1983; Kannel et al. 2007). These way susceptible organisms to pollution will decline and may disappear.

In a study conducted in these streams Moresco and Rodrigues (2014) detected that the effect of urbanization on periphytic diatoms in the urban stream was evident. This stream showed low richness values. On the other hand, in the rural stream, richness presented higher values. According to Moresco and Rodrigues (2014), the distinction in periphytic diatom assemblages among urban and rural streams were associated to the 

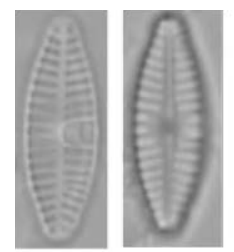

1

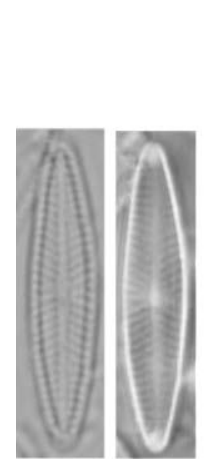

$14 \quad 15$
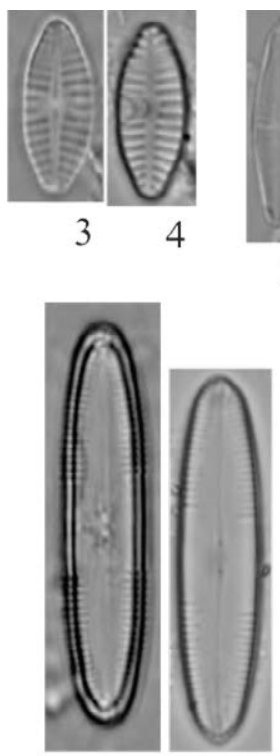

16

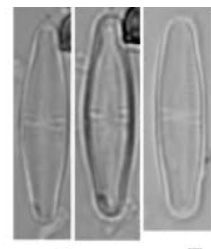

$\begin{array}{lll}5 & 6 & 7\end{array}$

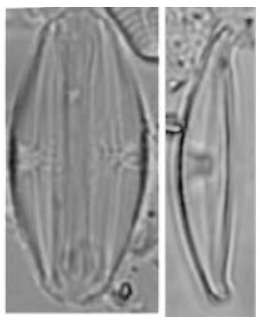

8

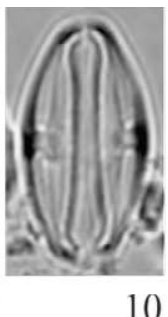

10

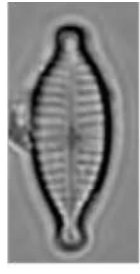

11

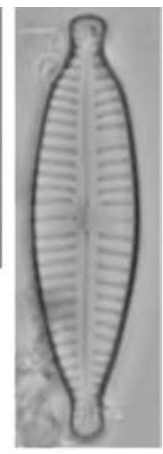

12

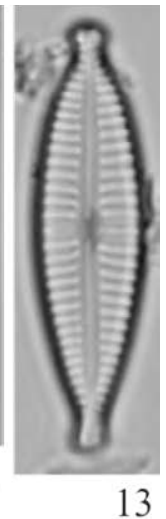

13

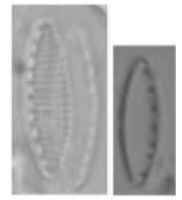

$18 \quad 19$

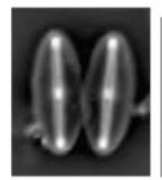

20

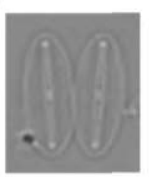

21

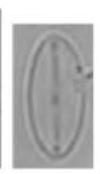

22

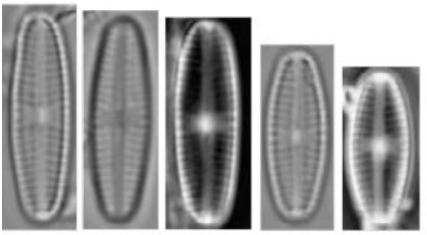

23-27

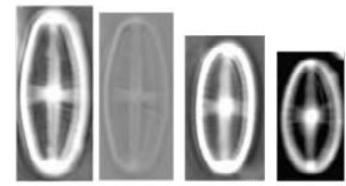

29-32

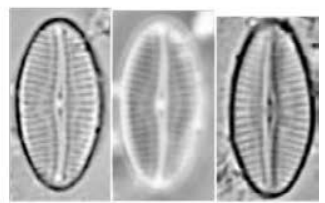

35-37

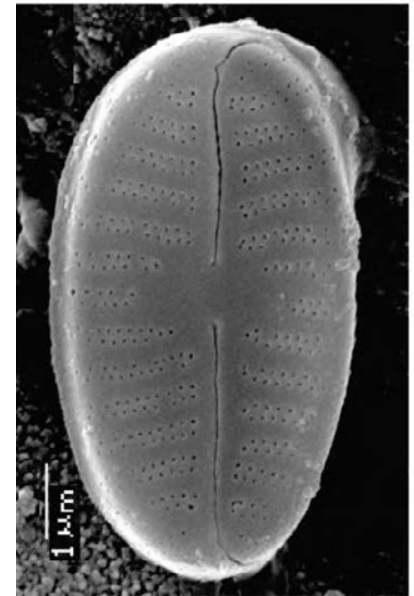

28

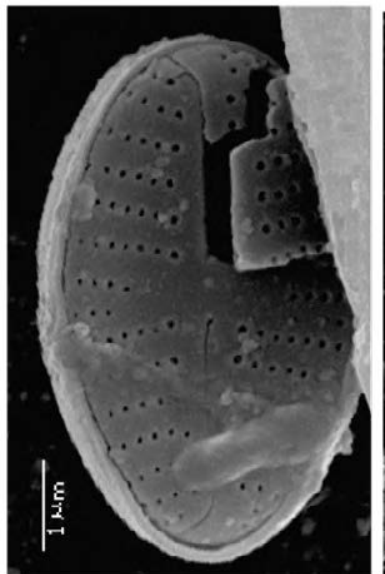

33

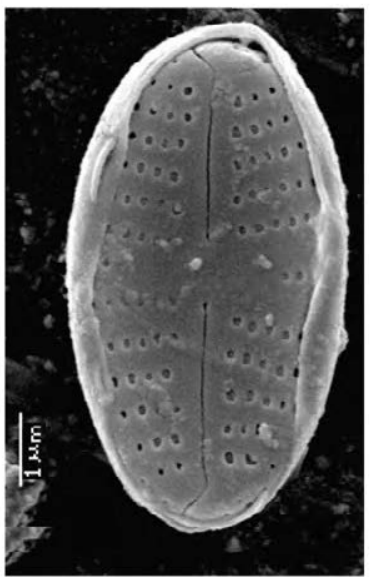

34

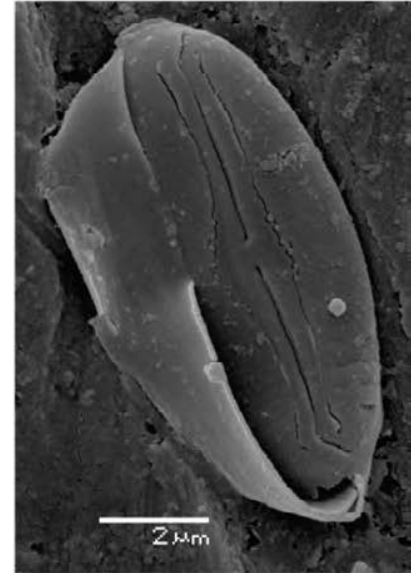

38

Figure 2. Taxa of diatoms. 1-4: Achnanthes lanceolata. 5-7: Achnanthidium minutissimum. 8-10: Amphora montana. 11-13: Gomphonema lagenula. 14-15: Navicula tenelloides. 16-17: Caloneis bacillum. 18-19: Nitzschia cf. inconspicua. 20-22: Mayamaea atomus var. permitis. 23-28: Sellaphora seminulum. 29-34: Eolimna minima. 35-38: Fallacia monoculata. Scale bar $=10 \mu \mathrm{m}$. For figures 28,33 and 34 the scale is $1 \mu \mathrm{m}$ and for figure 38 the scale is 2 $\mu \mathrm{m}$. Photos by C. Moresco and Priscila I. Tremarin.

increased conductivity and total nitrogen values found in urban stream and the upper dissolved oxygen, COD, $\mathrm{BOD}_{5}$, and flow in rural stream.

\section{ACKNOWLEDGEMENTS}

To the CNPq for the financial support to the project "Identification of possible bioindicators in urban aquatic ecosystems: the response of the groups of organisms to stress gradients" and to CAPES for scholarship. To PEA/ UEM and Nupélia/UEM for logistic support. To Priscila I. Tremarin and Thelma A.V. Ludwig for the help in identifying diatoms.

\section{LITERATURE CITED}

Ács, E., N.M. Reskone, K. Szabo, G. Taba and K.T. Kiss. 2005. Application of epiphytic diatoms in water quality monitoring of lake Velencei, Recommendations and Assignments. Acta Botanica Hungarica 47(3-4): 211-223. doi:10.1556/ABot.47.2005.3-4.1

Azim, M.E. and T. Asaeda. 2005. Periphyton structure, diversity and colonization; pp. 15-33, in: M.E. Azim, M.C.J. Verdegem, A.A. van Dam and M.C.M. Bederidge (eds.). Periphyton ecology, exploitation and management. Cambridge: CABI Publishing.

Brassac, N.M. and T.A.V. Ludwig. 2003. Fragilariaceae (Bacillariophyceae) de rios da bacia do Iguaçu Estado do Paraná, Brasil. Revista Brasileira de Botânica 26(3): 311-318.http://www.scielo. $\mathrm{br} / \mathrm{pdf} / \mathrm{rbb} / \mathrm{v} 26 \mathrm{n} 3 / 18950 . \mathrm{pdf}$

Delgado, C., I. Pardo and L. García. 2012. Diatom communities as 
indicators of ecological status in Mediterranean temporary streams (Balearic Islands, Spain). Ecological Indicators 15(1): 131-139. doi: 10.1016/j.ecolind.2011.09.037

Gómez, N. and M. Licursi. 2001. The Pampean Diatom Index (IDP) for assessment of rivers and streams in Argentina. Aquatic Ecology 35(2):173-181. doi: 10.1023/A:1011415209445

Kannel, P.J., S. Lee, S.R. Kanel, S.P. Khan and Y. Lee. 2007. Spatialtemporal variation and comparative assessment of water qualities of urban river system: a case study of river Bagmati (Nepal). Environmental Monitoring and Assessment 129(13):433-459. doi: 10.1007/s10661-006-9375-6

Kelly, M.G., A. Cazaubon, E. Coring, A. Dell'Uomo, L. Ector, B. Goldsmith, H. Guash, J. Hürlimann, A. Jarlman, B. Kawecka, J. Kwandrans, R. Laugaste, E.A. Lindstrom, M. Leitão, P. Marvan, J. Padisák, E. Pipp, J. Prygiel, E. Rott, S. Sabater, H. Van Dam and J. Vizinet, 1998. Recommendations for the routine sampling of diatoms for water quality assessments in Europe. Journal of Applied Phycology 10(2):215-224. doi: 10.1023/A:1008033201227

Krammer, K. and H. Lange-Bertalot. 1986. Bacillariophyceae: Naviculaceae; pp. 1-876, in: H. Ettl, J. Gerloff, H. Heynig and D. Mollenhauer (eds.). Süsswasserflora von Mitteleuropa 2(1): $1-876$.

Krammer, K. and H. Lange-Bertalot. 1988. Bacillariophyceae: Bacillariaceae, Epithemiaceae, Surirellaceae; pp. 1-596, in: H.Ettl, J. Gerloff, H. Heynig and D. Mollenhauer (eds.). Süsswasserflora von Mitteleuropa 2(2): 1-596.

Krammer, K. and H. Lange-Bertalot. 1991a. Bacillariophyceae: Centrales, Fragilariaceae, Eunotiaceae; pp. 1-576, in: H. Ettl, J. Gerloff, H. Heynig and D. Mollenhauer (eds.). Süsswasserflora von Mitteleuropa 2(3):1-576.

Krammer, K. and H. Lange-Bertalot. 1991b. Bacillariophyceae, Achnanthaceae. Kritische Ergänzungen zu Navicula (Linolatae) und Gomphonema; pp. 1-437, in:H. Ettl, G. Gärtner, J. Gerloff, H. Heynigand D. Mollenhauer (eds.). Süsswasserflora von Mitteleuropa 2(4): 1-437.

Krammer, K. 2002. Cymbella; pp. 1-584, in: H. Lange-Bertalot (ed.). Diatoms of Europe: diatoms of the European inland waters and comparable habitats 3:1-584.

Lange-Bertalot, H. 2001. Navicula sensu stricto-10 genera separated from Navicula sensu lato Frustulia; pp. 1-526, in: H. Lange-Bertalot, (ed.). Diatoms of Europe 2: 1-526.

Lobo, E.A., K. Katoh and Y. Aruga. 1995. Response of epilithic diatom assemblages to water pollution in rivers in the Tokyo Metropoli$\tan$ area. Freshwater Biology 34(1): 191-204.

Maack, R. 2002. Geografia Física do Estado do Paraná. Curitiba: Imprensa Oficial. 440pp.

Metzeltin, D., H. Lange-Bertalot and F. García-Rodriguez. 2005 Diatoms of Uruguay compared with other taxa from South America and elsewhere; pp. 1-736, in: H. Lange-Bertalot (ed.). Iconographia Diatomologica 15: 1-736.

Metzeltin, D. and H. Lange-Bertalot. 1998. Tropical diatoms of South America I. About 700 predominantly rarely know or new taxa representative of the Neotropical flora; pp. 1-695, in: H. LangeBertalot (ed.). Iconographia Diatomologica 5: 1-695.
Metzeltin, D. and H. Lange-Bertalot. 2007. Tropical diatoms of South America II. Special remarks on biogeographic disjunction; pp. 1-875, in: H. Lange-Bertalot (ed.). Iconographia Diatomologica 18: $1-875$.

Moreira-Filho, H. and I.M. Valente-Moreira. 1981. Avaliação taxonômica e ecológica das diatomáceas (Bacillariophyceae) epífitas em algas pluricelulares obtidas nos litorais dos Estados do Paraná, Santa Catarina e São Paulo. Boletim do Museu Botânico Municipal 47:1-17.

Moresco, C. and L. Rodrigues. 2014. Periphytic diatom as bioindicators in urban and rural streams. Acta Scientiarum 36(1): 67-78. doi: 10.4025/actascibiolsci.v36i1.18175

Odum, E.P. 1983. Ecologia. Rio de Janeiro: Guanabara Koogan. 613pp. Pan, Y.D., R.J. Stevenson, B.H. Hill, P.R. Kaufmann and A.T. Herlihy. 1999. Spatial patterns and ecological determinants of benthic algal assemblages in Mid-Atlantic streams, USA. Journal of Phycology 35(3): 46o-468. doi: 10.1046/j.1529-8817.1999.353046o.x

Patrick, R. 1961. A study of the numbers and kinds of species found in rivers of the Eastern United States. Proceedings of the Academy of Natural Sciences of Philadelphia113:215-258.

Patrick, R. and C.W. Reimer. 1966. The diatoms of the United States. Philadelphia: Academy of Natural Sciences, Monograph 13: 688 pp.

Peron, A.P., E.A. Canesin and C.M.V. Cardoso. 2009. Potencial mutagênico das águas do Rio Pirapó (Apucarana, Paraná, Brasil) em células meristemáticas de raiz de Allium cepa L. Revista Brasileira de Biociências 7(2):155-159. http://www.ufrgs.br/seerbio/ojs/index.php/rbb/article/view/1127

Potapova, M. and D.F. Charles. 2007. Diatom metrics for monitoring eutrophication in rivers of the United States. Ecological Indicators 7: 48-70. doi: 10.1016/j.ecolind.2005.10.001

Round, F.E., R.M. Crawford and D.G. Mann. 1990. The diatoms: biology and morphology of the genera. Cambridge, UK: Cambridge University Press. 747 pp.

Simonsen, R. 1974. The diatom plankton of the Indian Ocean Expedition of R/V "Meteor", 1964-65 "Meteor" Forschungsergbnisse. Reihe D-Biologie 19: 1-66.

Sonneman, J.A., C.J. Walsh, P.F. Breen and A.K. Sharpe. 2001. Effects of urbanization on streams of the Melbourne region, Victoria, Australia. II. Benthic diatom communities. Freshwater Biology 46(4): 553-565. doi: 10.1046/j.1365-2427.2001.00689.x

Wehr, J.D. and R.G. Sheath. 2003. Freshwater habitats of algae; pp. 11-57, in: J.D. Wehr and R.G. Sheat (eds.). Freshwater algae of North America: ecology and classification. San Diego: Academic Press.

Whitton, B.A. and M.G. Kelly. 1995. Use of algae and other plants for monitoring rivers. Australian Journal of Ecology 20(1): 45-56. doi: 10.1111/j.1442-9993.1995.tboo521.x

Author contributions: $\mathrm{CM}$ collected the data, $\mathrm{CM}$ identified the specimens, and CM and LR wrote the text.

Received: 11 June 2015

Accepted: 19 December 2015

Academic editor: Sandra Costa-Böddeker 